\title{
The challenges of model-based systems engineering for the next generation enterprise information systems
}

\author{
Milan Zdravković ${ }^{1} \cdot$ Hervé Panetto $^{2,3}$
}

Published online: 2 August 2017

(C) Springer-Verlag Berlin Heidelberg 2017

Transformation of the environment in which Enterprise Information Systems (EIS) are operating today is posing the new challenges to their modelling, design, implementation and use. One of the crucial circumstances of this transformation is removal of their traditional boundaries. The challenges arising from the increased requirements for flexibility in enterprise collaboration put a lot of pressure to the research on EIS integration and interoperability; they also urge for tools and approaches for rapid and active adaption of those EISs to the changing environment. The research and development of multi-agent systems paradigm are enabling EISs with multiple identities which are now representing enterprises in the digital space. The development of the Cyber-physical networks and the Internet of Things expanded the EIS scope, by introducing sensing and location-awareness aspects. The advances in cloud-based computing clearly established the distribution of the EISs' functional and storage capabilities as the default approach to their designs.

In a recently published position paper, based on the above circumstances, the Technical committee for Enterprise Integration and Networking of the International Federation of Automatic Control (IFAC TC5.3) proposed the concept of the Next Generation EIS (NG EIS), which is federated, omnipresent, model-driven, open, reconfigurable and aware. All these properties imply that the future NG EIS is inherently, natively interoperable.

One of the important effects of the foreseen properties, as EISs are becoming more complex, the gap between the problem-level software abstractions of the EIS

$\triangle$ Milan Zdravković

milan.zdravkovic@gmail.com

1 Faculty of Mechanical Engineering in Niš, University of Niš, Niš, Serbia

2 CRAN - UMR 7039 CNRS, Campus Scientifique, BP 70239,

54506 Vandoeuvre-Les-Nancy Cedex, France

3 CRAN, Université de Lorraine, Campus Scientifique, BP 70239,

54506 Vandoeuvre-Les-Nancy Cedex, France 
aspects on one side and their implementations, on another, will significantly increase. This increase will put a lot of pressure on the traditional Model-Based System Engineering (MBSE) theory and practice. In attempt to anticipate the directions for their future development, the special issue aims at addressing the implications arising from the foreseen evolution towards NG EIS.

While model based engineering is considered as one of the main interoperability enablers, it is still suffering from unresolved problems, due to some methodological and technological limitations. Zacharewicz et al. identified those problems in the following subfields: model alignment, model to system integration, reverse modelling, EIS and Human Resource (HR) models alignment and modelling validation indicators. The authors discussed about each of the groups of the problems in context of the future expectations from NG EIS. Finally, a conceptual framework for their resolution has been proposed.

In fact, vast majority of remaining papers in this SI are related to the first two of the above identified subfields, indicating that they are by far the most difficult to address. Next three papers, presented in the SI are directly related to model alignment.

Interoperability challenge is especially difficult at semantic level, where ad-hoc interoperations are expected to occur successfully. One possible solution is the design of formal model driven EISs, where ontologies are used to formally define EIS semantics, most desirably at different levels of abstractions. Such approach is proposed by Cverdelj-Fogaraši et al., who explored the benefits of using formal ontologies as meta-models at generic and domain levels for Document Management Systems.

Even if the semantic integration becomes possible with the use of formal models as the infrastructural basis of one EIS, the process of semantic mapping of two EISs' interfaces is still manual and thus, error prone. In their work, Vuković et al. aim at automating or semi-automating mapping and conflict resolution processes. Their proposed solution to semantic mapping is developer agnostic with respect to technical implementation of the involved EISs.

Model-to-model mapping and transformation is also the topic of interest of the work of Wang et al. However, instead of mapping the formal models, they go one step further and try first to effectively detect semantics in the existing models, while taking into account vast diversity of viewpoints used by those models, heterogeneity of existing modeling techniques and complexity of models, namely their volume and dynamics. Then, based on the identified semantics, they define model-to-model mappings and transformations, respectively.

Following two papers fall into the category of works which deal with the model to system integration.

Despite huge research efforts in enterprise architecture and modelling, the effectiveness of the existing approaches is still sometimes disputed. One of the yet unanswered research questions is: "How to effectively utilise enterprise models to generate enterprise software components?". Pazos and Chalmeta have proposed the framework, which follows the MDA approach, consisting of the well-defined methodology, a set of meta-models for CIM representation, modelling guide for 
using those meta-models, extraction algorithm to discriminate the part of the CIM model to use in transformation and a set of transformation rules.

Petrovic et al. addressed the similar problem but they proposed the solution to model to system integration issue in a very specific domain. Their work deals with complexity of data warehousing applications, namely design of complex and dynamic ETL (Extract-Transform-Load) processes, aiming at integration of different business data coming from different sources, transformation of that data to resolve structural and semantic conflicts; further translation into a form suitable for analysis, etc. For the formal specification of ETL processes, a set of new Domain Specific Languages (DSL) have been proposed. Then, the developed ETL-PL platform is used to design those processes and transform them into executable code for the specific application framework.

One of the challenges of MBSE theory and practice, not listed in the introductory work of Zacharewicz et al., is potential lack of expressivity of the existing models to deal with increasing complexity of the reality in which they are used. This challenge has been addressed by the following two papers.

Martinez et al. focused at business process models, one of the most dynamic aspects of one EIS operation. They argued that the existing models were still not very sensitive to the dynamics of the technology use in one organization. This is considered as a major issue, because the use of technology may significantly and positively affect the performance of the process itself and even modify the workflow. Hence, they proposed a systematic process for integration of business processes and technologies at conceptual level, by using $i *$ framework.

Venkatesan and Sridhar proposed to bring further extensions to existing process models. In specific they extended BPEL framework with novel architectural primitives, aiming at facilitating migration of code and speech-act based component orchestration/application composition.

The SI concludes with the presentation of research work by Vallejo et al. They introduced a methodology for designing and assessing the manufacturing enterprise operations, from the project management perspective. The methodology is based on Program Logic Models and System Dynamics. 